\title{
Harvesting the Blue Ocean Wave Energy with a Circular Electromagnetic Generator Prototype
}

\author{
Kerui Yang \\ Edina High School, 6754 Valley View Rd, Edina, MN, 55439, U.S.A.; kerui2006@gmail.com
}

\begin{abstract}
The oceans provide enough sustainable wave energy to power human civilization. However, due to the inefficiency and ineffectiveness of current technologies, most of this energy remains unharvested. My research aimed to fabricate and test a new, inexpensive, portable, and efficient device to capture wave energy. The Circular Electromagnetic Generator (CEG) has a magnetic sphere traveling within a toroid tube coiled with copper wire. Electricity is generated as waves propel the magnet to roll. A dumbbell-shaped transducer was created to understand how the size and speed of the magnetic sphere would affect the electrical output. It was observed that larger magnetic spheres moving at higher speeds produced greater voltage outputs. A CEG unit was fabricated and a Wave and Rocking Shaker was used to simulate wave movements. The experimental results showed that the CEG can capture the kinetic energy of small waves since small tilt angles and low rocking speeds induced the CEG to produce electricity. A waterproof CEG unit prototype composed of a CEG unit, full wave rectifier, smoothing capacitor, and LED was able to float and generate electricity as the water moved. Additionally, a CEG farm was proposed and modeled to capture large amounts of wave energy.

KEYWORDS: Energy; Sustainable Ocean Wave Energy; Physical Energy; Circular Electromagnetic Generator.
\end{abstract}

\section{- Introduction}

The United States utilizes 21\% of the world's total energy and relies heavily on nonrenewable fossil fuels (oil, coal and natural gas) and nuclear energy, while renewable energy makes up only $11 \%$ of energy that we use. ${ }^{1,2}$ Burning fossil fuels releases massive amounts of carbon dioxide into the atmosphere and increases the rate of climate change. Hydraulic fracturing for natural gas and oil requires millions of gallons of water that depletes water supplies for nearby communities, releases methane and wastewater back into the environment, and increases the possibilities for oil spills and earthquakes. ${ }^{3}$ Nuclear power is a dangerous as well as expensive resource and may lead to nuclear meltdowns, nuclear waste, and radiation leakage. ${ }^{4}$ Due to their limited supply and detrimental effects to the environment these energy resources are not sustainable, necessitating an increased reliance on green energy.

Renewable energy is generated from resources including solar radiation, wind, waves, biomass, and geothermal heat. These energy sources are constantly replenished by nature, so society only needs to focus on developing energy-harnessing and conversion technologies without much concern for the energy source depletion. Furthermore, renewable energies impose minimal pollution to the environment because there is no byproduct or waste in the power generation process. In recent years, renewable energy-harnessing technologies have led to tremendous progress in the awareness and adoption of renewable energy.

Oceans cover more than $70 \%$ of the Earth's surface, making them an important renewable energy source. ${ }^{5}$ There are five different types of ocean energy: tidal, water wave, ocean current, temperature gradient, and salinity gradient. ${ }^{5}$ Water wave energy refers to the kinetic and potential energy originating from the movements of surface ocean waves. Open oceans can provide up to 20-30 terawatts (TW) of power annually, which is greater than the amount consumed by human society (roughly 15 TW annually). ${ }^{5}$ Compared to solar and wind energy, water wave energy depends much less on the season, climate, and temperature, making it a more predictable resource with higher power density. ${ }^{6}$ Furthermore, the U.S. has many coastlines $(95,471 \text { miles })^{7}$ with high wave power and more than one third of the world's wave energy companies. These benefits give the U.S. a tremendous advantage in leading the future development and adoption of ocean wave energy. ${ }^{6}$

There are four main categories of existing ocean wave energy technologies: terminator devices, attenuators, point absorbers, and overtopping devices. ${ }^{8}$ Terminator devices utilize an oscillating water column to drive a turbine for electricity production. Attenuators have multiple long segments connected to hydraulic pumps and other converters to generate power. Point absorbers are floating buoys linked to the seafloor that harness the rising and falling wave movements to drive electromagnetic energy convertors. Overtopping devices utilize the buildup and release of water pressure inside reservoirs to turn hydro turbines. ${ }^{9}$ All of these devices are usually located nearshore or onshore.

Since these devices are usually connected to the seabed and located onshore or nearshore, they cannot be used in the open ocean. However, the power collected globally from areas near the shoreline has been estimated to be $2-3 \mathrm{TW}$, only a tenth of the possible power collected on the open ocean. ${ }^{10}$ All of the aforementioned devices utilize electromagnetic generators to 
produce electricity. These generators are bulky and have to be mounted onto the seabed or float with support from a separate platform or buoy. For this reason, they are expensive and difficult to install. ${ }^{10,11}$ Due to the challenges and downfalls posed by these devices, harnessing wave energy is still not popular. New technologies and devices are critical to increasing the use of wave energy. Recently, Triboelectric Nanogenerators were explored based on the conjugation of tribo-electrification and electrostatic induction. ${ }^{12}$ However, this technology has limited practical applications due to its output of high voltage and low current.

\section{Newly Designed Circular Electromagnetic Generators}

The goal of this project was to fabricate and test an inexpensive, compact, portable, and efficient device to capture ocean wave energy. The proposed Circular Electromagnetic Generator (CEG) utilizes the electromagnetic effect to produce electricity by rolling a magnetic sphere inside a closed, hollow, ring-shaped tube that is coiled with copper wire. This ring is housed inside a sealed waterproof container that enables floating. Additionally, a CEG farm can be created by linking thousands of these devices together and harnessing a great amount of open ocean wave energy. The CEG farm can be easily installed and is workable onshore, nearshore, and in open ocean.

The goal of this research was: (1) to design and build a novel, effective, and economical device to harness wave energy; (2) to investigate the various factors affecting the electrical output of this device; (3) to create a working prototype of this device; and (4) to design a CEG farm for practical applications

\section{- Results and Discussion Geometrical Design for Optimal Magnetic Sphere Rolling}

This research aimed to create a device to generate electricity by having a magnetic sphere roll within a tube wrapped with copper wires as the waves move. The magnetic sphere is expected to move freely and avoid crashing into the sides of the tube in response to wave movements from all directions so that energy harvesting and the durability of the device can be maximized.
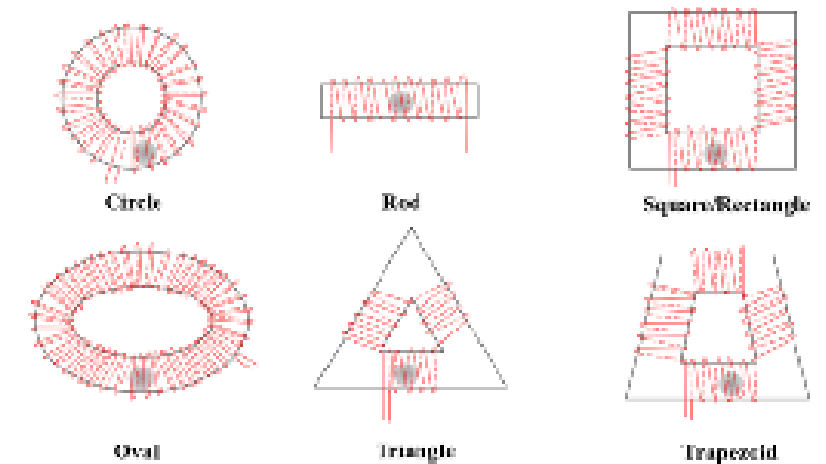

Trapexeid
With these requirements in mind, six different designs were considered (Figure 1). For the rod, square/rectangle, triangle, and trapezoid shapes, the magnetic sphere cannot roll freely in response to waves moving in certain directions. Therefore, the device cannot harness the wave's energy in some directions. In contrast, the magnetic sphere in the oval and circle-shaped devices can roll freely in response to waves moving in any direction. However, the oval shape possesses two tight curbs. The tight curbs, corners, and ends can cause the sphere to collide with the sides of the tubes. During these collisions, the sphere loses kinetic energy and thus decreases the electrical output. After repeated collisions, the tube and the magnetic sphere can become damaged, shortening the device's lifetime. Ultimately, the circular design is optimal because its uniform curvature results in greater kinetic energy preservation and maximizes durability. (a)

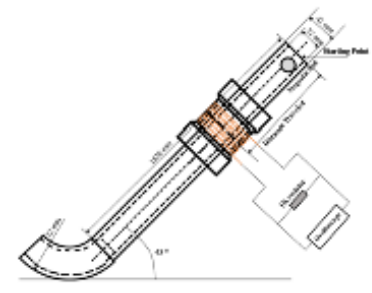

(b)

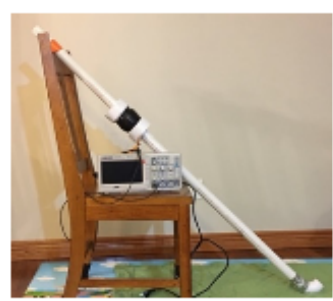

Figure 2. The experimental set up for the dumbbell shaped transducer. (a) Schematic diagram. (b) Picture of experimental set up.

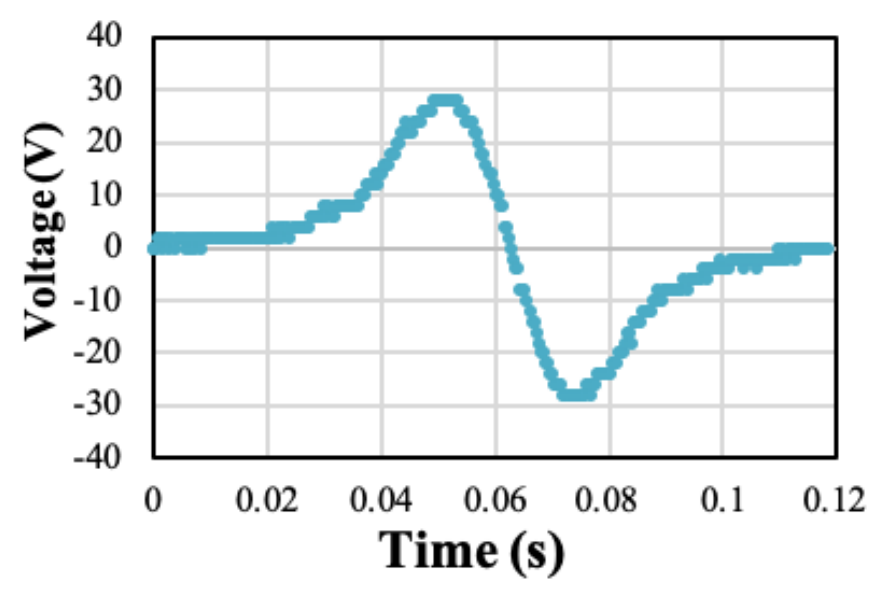

Figure 3. Plot for the voltage output as the 1.26 " magnetic sphere rolled through the copper wire coils of the dumbbell transducer.

\section{Electrical Output of the Dumbbell Shaped Transducer}

As the sphere rolls through a tube coiled with copper wire an electrical output is generated. The apparatus in Figure 2 was constructed in order to test this hypothesis. The center of the transducer was anchored $127 \mathrm{~cm}$ away from the top end of the PVC pipe. A 1.26" magnetic sphere was set gently and released at the top entrance of the PVC pipe. As the sphere rolled through the copper wire coils the digital oscilloscope recorded the electrical output as shown in Figure 3. The oscillating electrical output observed in Figure 3 appeared consistently across all trials. The positive electrical output is created when the sphere approaches the copper wires and increases the magnetic flux. The sphere's departure from the coiled copper wire

Figure 1. Diagram of the different designs for the device 
decreased the magnetic flux, producing a negative electrical output.

As shown in Figure 4, the highest positive voltage output varied greatly throughout the 20 experiments. This electrical output variation is due to the changing magnetic field directions as the magnetic sphere rolled down the PVC pipe. In order to maintain a constant magnetic field direction, two $3 / 8$ " magnetic spheres were attached to opposite sides of the 1.26 " magnetic sphere so the spheres would slide down the PVC pipe instead of rolling.

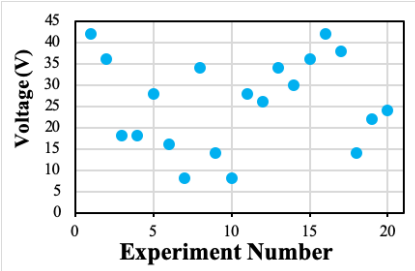

Figure 4. Plot for the highest positive voltage output resulting from rolling a 1.26" magnetic sphere down the PVC pipe 20 times.

As shown in Figure 5, comparable highest positive voltages were produced throughout the six experiments. Additionally, the transducer was utilized to investigate how the magnetic sphere's size and sliding speed affected the voltage output. For the following experiments, either two magnetic spheres of the same size (3/4", 5/8", 1/2", 3/8") were attached together or two $3 / 8$ " magnetic spheres were attached to opposite sides of the 1.26 " magnetic sphere.

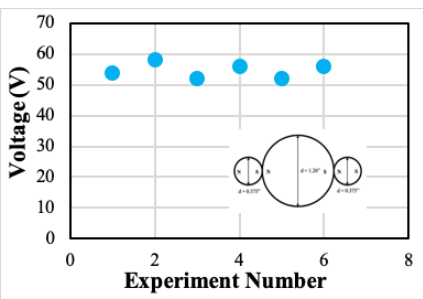

Figure 5. Plot for the highest positive voltage output resulting from sliding the sphere down the PVC pipe. Note: two 3/8" magnet spheres attached to opposite sides of the 1.26 " magnetic sphere.

As the total volume of the two equivalent magnetic spheres increased, the highest positive voltage also increased, shown in Figure 6. This phenomenon can be explained by Faraday's Law of Magnetic Induction where voltage is proportional to the

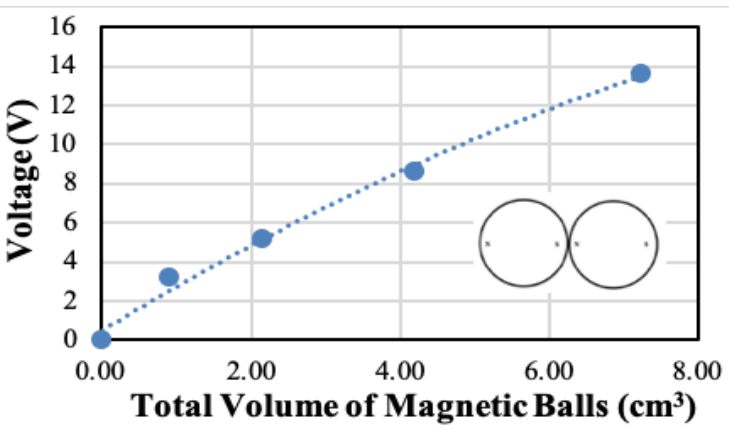

Figure 6. Plot of the highest positive voltage versus the total volume of the two equivalent magnetic spheres attached together. Note: two equivalent magnet spheres (3/4", $5 / 8$ ", 1/2", 3/8") attached together. change in magnetic field strength (which is proportional to the sphere's volume since they are made from the same material).

In Figure 7, it was shown that longer distances traveled by the magnetic spheres resulted in greater highest positive voltage outputs. The sliding speed of the magnetic spheres passing through the magnetic wire loops can be calculated according to the following equation $v=\sqrt{ }(\sqrt{ } 2 \mathrm{gL})$. As shown in Figure 8 , as the sliding speed increased, the highest positive voltage signal also increased linearly.

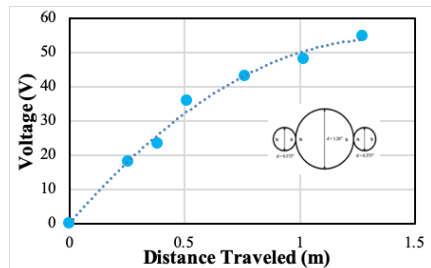

Figure 7. Plot of the highest positive voltage versus the distance traveled. Note: two $3 / 8$ " magnetic spheres attached to opposite ends of the 1.26 " magnetic sphere.

\section{CEG Unit Performance}

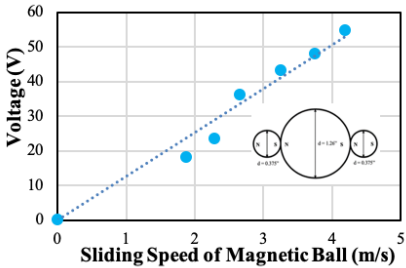

Figure 8. Plot of the highest positive voltage versus the sliding speed. Note: two 3/8" magnetic spheres attached to opposite ends of the 1.26 " magnetic sphere.

The movement of ocean waves was simulated using a Wave and Rocking Shaker. The set-up for the experiment is shown in Figure 9. A 1.26" magnetic sphere was used, and the rocking speed and tilt angle of the shaker was set to $50 \mathrm{rpm}$ and 10 degrees respectively. The CEG unit produced a continuously oscillating electrical output. After adding a full wave rectifier, the negative electrical output was converted into a positive electrical output, as shown in Figure 10. A subsequent addition of a $100 \mathrm{uF}$ smoothing capacitor converted the rippled output into a smoother electrical output.

The effects of rocking speed and tilt angle on the electrical output were investigated. The digital oscilloscope continuously recorded the electrical output for 30 seconds and the average electrical output was used for analysis. As shown in Figures 11 and 12, low rocking speeds and small tilt angles yielded an electrical output. This signifies that the CEG unit can capture kinetic energy even from small waves. (a)

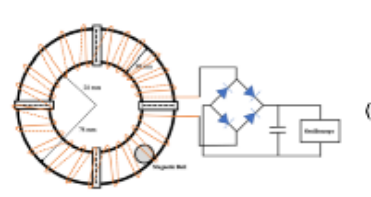

(b)

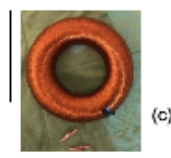

(c)

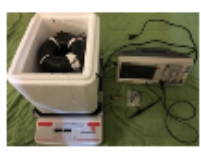

Figure 9. a) Schematic diagram of the experimental set up for the electrical output of CEG unit. (b) Picture of the CEG unit. (c) Picture of the experimental set up for the electrical output of CEG unit. 
Waterproof CEG Prototype for Wave Energy Harvesting

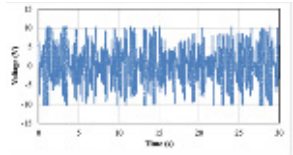

(a)

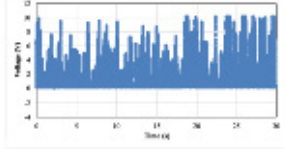

(b)

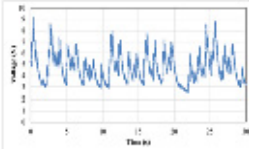

(c)
Figure 10. Plot of the voltage output for CEG unit. (a) No accessory. (b) A full wave rectifier added. (c) A full wave rectifier and smoothing capacitor added. Note: the speed and tilt of the Wave and Rocking Shaker was set to $50 \mathrm{rpm}$ and 10 degrees, respectively.

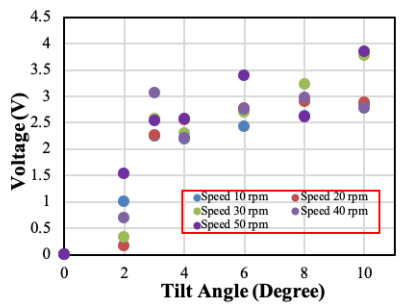

Figure 11. Plot of the average voltage versus the tilt angle for five different rocking speeds.

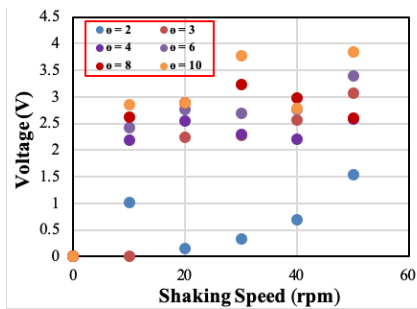

Figure 12. Plot of the average voltage versus rocking speed for the six different tilt angles.

The ocean is high in salt content and humidity, so a closed, plastic container was chosen to house the CEG as it is waterproof and enables the CEG to float above the water's surface. The total mass of the CEG prototype was $360 \mathrm{~g}$. Placing the CEG prototype in a swimming pool proved the CEG could float above the water's surface. As the water moved, the LED lit up. This device was able to generate about $2 \mathrm{~V}$ of voltage to power a $0.06 \mathrm{~W}$ LED light bulb. This confirms the feasibility of the CEG unit as an effective device to capture water wave energy.

The total cost to build this waterproof CEG prototype was $\$ 27.58$. Because resources were not bought in bulk the prototype is considerably more expensive than one unit in mass production. Therefore, for this cost comparison, the cost of a CEG unit in large scale production is scaled down to ten percent of the original cost, $\$ 2.80 .{ }^{13}$ This cost is comparable to a single Duracell Coppertop D Alkaline Battery (\$9.99 for a 4 pack on Amazon).

\section{CEG Farm for Wave Energy Harvesting}

As illustrated in Figure 13, thousands of these waterproof CEG devices can be linked together via flexible tubing and floating frames to form a CEG farm. Each individual CEG unit can be used as a power source for electronics while a large scale CEG farm can function as a large-scale electricity generator. The power from the CEG farm can be transferred to power plants or the electric grid on land.

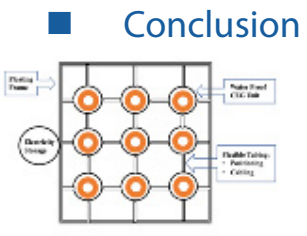

(a)

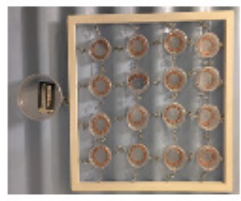

(b)

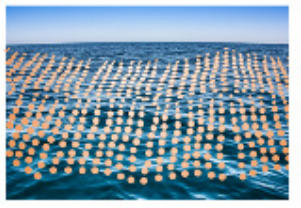

(c)
Figure 13. (a) Schematic diagram for the proposed CEG farm structure. (b) Picture of the CEG farm model. (c) Illustration of a large scale CEG farm.

The CEG is an effective device to produce electricity by having water waves drive a magnetic sphere to roll inside a toroid tube coiled with enameled copper wire. The transducer experiment showed that larger magnetic spheres moving at higher speeds produced greater voltage outputs. A Wave and Rocking Shaker was used to simulate the movement of ocean waves, and small tilt angles and low rocking speeds were able to induce the CEG to produce electricity. This demonstrated that the CEG unit can capture the kinetic energy even from small waves. Then, a waterproof CEG prototype unit was fabricated and successfully generated electricity in the presence of water waves. Finally, a CEG farm was proposed to capture large amounts of wave energy and a model was created.

\section{- Methods and Materials}

Resistors were from Elegoo. $5 \mathrm{~mm}$ round 2 Pin LED was from Lead Star. $100 \mathrm{uF}$ electrolytic capacitors were from Electronics-Salon. 1A 100V 59-10 standard diode was from On Semiconductors. 13200 Gauss grade N42 neodymium magnetic spheres with diameter of 1.26 ”, 1", 3/4", 5/8", 1/2" and 3/8" were from Applied Magnets. 24, 30 and 31 AWG magnet enameled copper wires were from Remington Industries. 1-1/2" 90'street elbow PVC DWV was from NIBCO. 1 1/4" DWV PVC Type $1 \mathrm{SCH} 40$ Pipe was from Charlotte Pipe. 2" x 1-1/2" PVC DWV reducing coupler was from NIBCO. 1-1/4 in. $\times 5$ ft. furniture grade Sch. 40 PVC Pipe was from Formufit. Plastic container was from Keeper Flexible Seaware. Plastic tube (Brilliant Rock-a-Stack) was from Fisher-Price. The Wave and Rocking Shaker SHRK04DG was from OHAUS. The rocking speed varied from 0-50 rpm and the tilt angle varied from 0 to 10 degrees. SDS1072CM Digital Oscilloscope (7" TFT-LCD Display Bench-Top, 70MHz) was from Siglent.

\section{Fabrication of the Dumbbell Shaped Transducer}

A $62 \mathrm{~mm}$ long 1.25 " PVC pipe was inserted and glued between the narrow ends of two 2"x 1-1/2"PVC DWV reducing couplers (Figure 14). $2.5 \mathrm{lb} .(60,198 \mathrm{~cm})$ of 24 AWG enameled copper wire was evenly and tightly coiled around the pipe. The wire was coiled 2,813 times around the tube with each loop having a circumference of $21.4 \mathrm{~cm}$. The two ends of the wire were connected to a $1 \mathrm{~K} \mathrm{ohm}$ resistor and the voltage of the resistor was measured by a digital oscilloscope. 
(a)

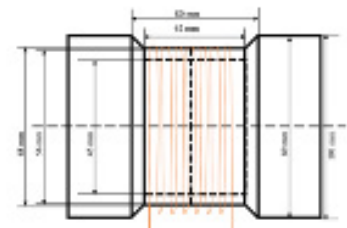

(b)

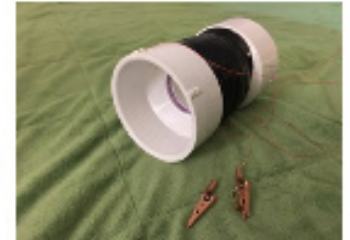

Figure 14. Dumbbell shaped transducer. (a) Schematic diagram. (b) Picture of experimental set up.

\section{Measuring the Electrical Signal from the Movement of a} Magnetic Sphere through the Dumbbell Shaped Transducer

The 1 1/4" DWV PVC pipe was placed at an angle of $45 \mathrm{de}-$ grees with respect to the ground. This PVC pipe was inserted through the transducer and the transducer was anchored using tape (Figure 2). The center of the transducer was positioned at various distances from the top of the PVC pipe. The magnetic sphere was released gently at the top entrance of the PVC pipe. The distance traveled (L) by the sphere was defined as the distance between the top end of the PVC pipe and the center of the transducer.

Assuming that the frictional force between the magnetic sphere and the PVC pipe is negligible and setting the initial magnetic sphere sliding speed to $0 \mathrm{~m} / \mathrm{s}$, the sliding speed of the magnetic sphere after traveling a distance $\mathrm{L}$ is equal to $\sqrt{ }(\sqrt{2} \mathrm{gL})$, where $\mathrm{g}$ is the gravitational acceleration $\left(9.8 \mathrm{~m} / \mathrm{s}^{2}\right)$. After releasing the magnetic sphere at the top end of the PVC pipe, the digital oscilloscope started to record the electrical signal produced by the magnetic sphere traveling through the dumbbell shaped transducer. For each different L, the electrical signal was measured three times and the highest positive voltage average was taken.

\section{Fabrication of the CEG Unit}

A 1.26" magnetic sphere was placed into a circular tube formed by gluing together four $1.5^{\prime \prime} 90^{\circ}$ street elbow PVC pipes (Figure 9). After the glue had solidified, 1 lb. $(95,464$ $\mathrm{cm}$ ) of 30 AWG enameled copper wire was evenly and tightly coiled around the pipe. The wire was coiled 6,080 times around the tube with each loop having a circumference of $15.7 \mathrm{~cm}$. The two ends of the wire were connected to a full wave rectifier made up of four 1A 100V 59-10 standard diodes. A $100 \mathrm{uF}$ capacitor was then added to the circuit.

\section{Measuring the Electrical Output from the CEG Unit}

The CEG unit was placed inside a Styrofoam container, which sat on the metal tray of the Wave and Rocking Shaker and anchored using tape. The distance between the CEG unit and the tray surface was adjusted to $17.8 \mathrm{~cm}$ by foam padding in order to nullify the attraction between the magnetic sphere and the metal tray.

The tested rocking speeds were $10 \mathrm{rpm}, 20 \mathrm{rpm}, 30 \mathrm{rpm}, 40$ $\mathrm{rpm}$, and $50 \mathrm{rpm}$. The tilt angles used for experimentation were $2^{\circ}, 3^{\circ}, 4^{\circ}, 6^{\circ}, 8^{\circ}$, and $10^{\circ}$. After stabilizing the tested rocking speed and tilt angle, the electrical output was recorded three times using a digital oscilloscope. All combinations of rocking speeds and tilt angles were tested.

\section{Fabricating a Waterproof CEG Prototype}

A 1" magnetic sphere was placed into a hollow plastic ring (Brilliant Rock-a-Stack) through a cut slit that was later taped shut (Figure 15). $0.25 \mathrm{lb}$. (30,906 cm) of 31 AWG enameled copper wire was evenly and tightly coiled around the ring. The wire was coiled 3,091 times with each loop having a circumference of $10 \mathrm{~cm}$. The two ends of the wire were connected to a full wave rectifier made up of four $1 \mathrm{~A} 100 \mathrm{~V}$ 59-10 standard diodes. A $100 \mathrm{uF}$ capacitor was then added to the circuit.

The CEG unit was placed into a plastic container $(10 \mathrm{~cm}$ tall by $15 \mathrm{~cm}$ wide). Foam spacers were used to secure around and above the CEG unit. The full wave rectifier, capacitor, and LED were placed above the foam spacer. The container was sealed tightly with a cap. The total mass of the waterproof CEG prototype was $360 \mathrm{~g}$. (a)

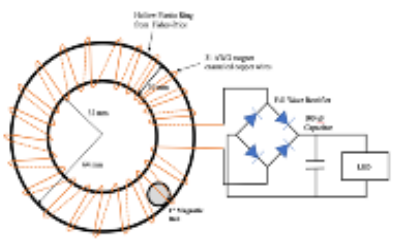

(c)

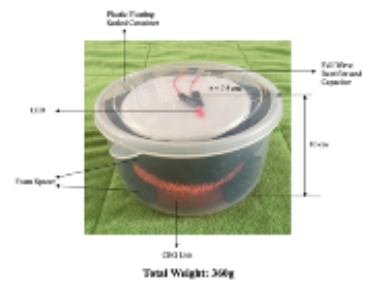

(b)

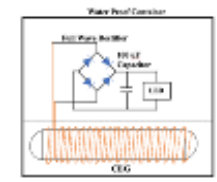

(d)

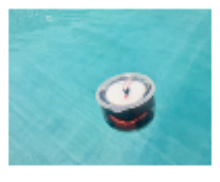

Figure 15. Schematic diagram of the waterproof CEG prototype unit. (b). Schematic diagram of the side view for the prototype. (c) Picture for the waterproof CEG prototype. (d) Picture of the prototype floating in a swimming pool.

\section{Acknowledgements}

Thank you to my parents and supervisor, as well as Genius Olympiad for giving me this opportunity.

\section{References}

1. U.S. Energy Information Administration. U.S. Energy Facts Explained. https://www.eia.gov/energyexplained/?page=us_energy home (accessed November 15, 2018).

2. Vox. American Energy Use, In One Diagram. https://www.vox.com/energy-and-environment/2017/4/13/ 15268604/american-energy-one-diagram (accessed November 17, 2018).

3. Investopedia. What are the Effects of Fracking On The Environment?. https:/www.investopedia.com/ask/answers/011915/ what-are-effects-fracking-environment.asp (accessed November 23, 2018).

4. Greenpeace. Nuclear Energy. https://www.greenpeace.org/usa/global-warming/issues/nuclear/ (accessed November 15).

5. Wang, Z. L.; Jiang, T.; Xu, L. Toward the Blue Energy Dream by Triboelectric Nanogenerator Networks. Elsevier. 2017, 39, 9-23.

6. Lehmann, M.; Karimpour, F.; Goudey, C.; Jacobson, P.; Alam, M. R. Oceans Wave Energy in the United States: Current Status and Future Perspectives. Elsevier. 2017, 74, 1300-1313.

7. National Ocean and Atmospheric Administration. How long is the U.S. shoreline? https:/oceanservice.noaa.gov/facts/ shorelength.html (accessed Nov. 2)

8. Bureau of Ocean Energy Management. Ocean Wave Energy. 
https://www.boem.gov/Ocean-Wave-Energy/

(accessed November 20).

9. Lim, S. J. Wave Energy Converters. Stanford University. 2013.

10.Chen. J.; Yang, J.; Li, Z. L.; Fan, X.; Zi, Y. L.; Jing, Q. S.; Guo,

H. Y.; Wen, Z.; Pradel, K. C.; Niu, S. M.; Wang, Z. L.

Networks of Triboelectric Nanogenerators for Harvesting Water

Wave Energy: A Potential Approach toward Blue Energy.

ACS Nano. 2015, 30, A-H.

11.Xu, L.; Jiang, T.; Lin, P.; Shao, J. J.; He. C.; Zhong, W.; Chen, X. Y.; Wang,

Z. L. Coupled Triboelectric Nanogenerator Networks for Efficient Water

Wave Energy Harvesting. ACS Nano. 2018, 40, A-J.

12.Vista Industrial Products Inc. Prototype vs. Production vs. Mass

Production. http://www.vista-industrial.com/blog/ prototype-vs-

production-vs-mass-production/ (accessed Nov. 2).

\section{- Authors}

Kerui Yang has participated in her local science fair since seventh grade. Her research has spanned topics from harvesting wind and ocean energy to biological studies, such as DNA sequencing and glucose and fluoride sensing. 